\title{
Ecto-5'-nucleotidase (CD73) inhibits dorsal root ganglion neuronal apoptosis by promoting the Ado/cAMP/PKA/CREB pathway
}

\author{
MINGHAO SHAO $^{1}$, CHAOJUN ZHENG $^{1}$, XIAOSHENG MA $^{1}$ and FEIZHOU LYU ${ }^{1,2}$ \\ ${ }^{1}$ Department of Spine Surgery, Huashan Hospital, Fudan University; ${ }^{2}$ Department of Orthopedics, \\ Shanghai Fifth People's Hospital, Fudan University, Shanghai 200040, P.R. China
}

Received September 4, 2020; Accepted April 21, 2021

DOI: $10.3892 / \mathrm{etm} .2021 .10809$

\begin{abstract}
Spinal cord injury (SCI) is a serious affliction that can lead to insufficient blood supply to the spinal cord, resulting in nutrient and energy deficiency in nerve cells such as neurons. In the present study, a spinal cord injury mouse model was constructed using wild-type (WT) and ecto-5'-nucleotidase (CD73) $)^{-/-}$mice. The results of TUNEL and immunofluorescence assays indicated that the apoptosis of neurons in $\mathrm{CD}_{73^{--}}$mice was increased after spinal cord injury. Dorsal root ganglion (DRG) neurons from WT and $\mathrm{CD}^{-/-}$mice were cultured in low glucose and hypoxic conditions to simulate the effects of spinal cord injury on neurons. Subsequently, a western blot assay was used to detect the expression of CD73, caspase-3 and Bcl-2. Flow cytometry was used to detect cell apoptosis and the corresponding kits were used to detect changes in lactate dehydrogenase (LDH), superoxide dismutase (SOD), malondialdehyde (MDA), reactive oxygen species (ROS), adenosine triphosphate (ATP) and cell activity. The results revealed that the apoptosis level of CD73-overexpressing DRG neurons was decreased under anoxia and glucose deficiency. The release of LDH, MDA and the production of ROS in CD73 DRG neurons was decreased, while the synthesis of ATP, the activity of SOD and cell activity increased after hypoxia-hypoglycemia treatment. Additional cellular studies demonstrated that blocking the expression and hydrolase activity of CD73 with $\alpha, \beta$-methylene ADP (APCP) could counteract the protective effect of CD73 on neuronal apoptosis, while adenosine (Ado) could rescue the increased apoptosis caused by CD73 deletion. In addition, the $\mathrm{cAMP} /$ protein kinase $\mathrm{A}$ (PKA)/cAMP response element-binding protein (CREB) signaling pathway was also positively regulated by $\mathrm{CD} 73$ and Ado. In conclusion, CD73 could inhibit DRG neuronal apoptosis by promoting the Ado/cAMP/PKA/CREB pathway.
\end{abstract}

Correspondence to: Professor Feizhou Lyu, Department of Spine Surgery, Huashan Hospital, Fudan University, 12 Wulumuqi Middle Road, Shanghai 200040, P.R. China

E-mail: zytgdily@126.com

Key words: spinal cord injury, dorsal root ganglion neurons, CD73, adenosine, cAMP

\section{Introduction}

Spinal cord injury (SCI) is a serious affliction mainly caused by trauma. Because nerve tissue is difficult to repair, SCI can lead to a great disorder of sensory and motor functions below the injury plane, and even cause life-long pain in patients (1). In recent years, with the increase in traffic and industrial accidents, the incidence of acute SCI has been increasing in China yearly (2-4). At present, except for the surgical relief of compression, there is no ideal treatment method for acute $\mathrm{SCI}$ (5). Therefore, it is necessary to further explore the mechanism of the pathological process of acute SCI, discover and develop appropriate drugs to block its pathological process and promote the recovery of nerve function.

The pathophysiological mechanism of SCI is complicated and includes primary and secondary injury stages. Primary injury involves cell death or degeneration induced by the sequential activation of local and systemic inflammatory responses and post-injury adaptation of primary injury (6). Secondary injury commences a short time after the trauma and lasts for an extended period of time, which can cause additional degeneration or death of neurons, and is no less harmful to the nervous system than primary injury (7). After acute SCI, vascular injury leads to a series of changes in spinal cord blood supply, including abnormal spinal cord blood supply and perfusion, eventually leading to hemorrhage, ischemia and reperfusion injury, and the death of neurons and glial cells $(8,9)$. The death of nerve cells is mainly caused by apoptosis, which is considered to be a main inducer of nervous system diseases (10). Due to the importance and non-reproducibility of neurons, it is particularly important to prevent neuronal apoptosis resulting from spinal cord injury (11). A previous study revealed that energy metabolism disorders caused by SCI ultimately induce neuronal apoptosis (12). However, the exact mechanism remains unclear.

Ecto-5'-nucleotidase (CD73), a nucleotide enzyme that is attached to the plasma membrane, mediates the production of adenosine (Ado) to control the production of purine nucleotides and thus the signaling of nucleotides $(13,14)$. CD73 can mediate the binding of Ado and its receptor to form cAMP which can reduce the permeability of the blood-brain barrier (14). In addition, Ado can protect the ischemic brain and spinal cord as an endogenous neuroprotective factor $(15,16)$. Our previous study demonstrated that $\mathrm{CD} 73$ can promote the M2 polarization 
of microglia, weaken the inflammatory response and inhibit caspase-3 gene expression after SCI, indicating that CD73 has a protective effect on SCI (17). However, whether there is a protective effect of CD73 on neurons remains to be elucidated. Therefore, the relationship between CD73 and the apoptosis of injured spinal cord neurons was studied through a series of experiments to clarify the protective mechanism of CD73 in SCI.

\section{Materials and methods}

Animals. C57BL/6 CD73 knock out (CD73-/) male mice were kindly gifted by Professor Thompson, Oklahoma Medical Research Foundation (Oklahoma City, USA). The Shanghai SLAC Laboratory Animal Co., Ltd., supplied the wild-type (WT) male C57BL/6 mice. A total of 10 CD73 knockout mice and 10 wild-type mice were used for subsequent experiments. Each mouse was $\sim 3$ months old and weighed $\sim 30$ g. All surgical procedures and experimental protocols in the present study were performed in accordance with standard guidelines approved by the Ethics Committee of Experimental Research, Shanghai Medical College, Fudan University (Shanghai, China). The approval number is 2019-Huashan Hospital-JS-104.

Construction of spinal cord injury model mice. Each of the 10 male WT C57BL/6 mice and $10 \mathrm{CD} 73^{-/-} \mathrm{C} 57 \mathrm{BL} / 6$ mice were fed under temperature $28^{\circ} \mathrm{C}$, relative humidity $40 \%$, ventilation reach 20 times/h, light/dark cycle durations is $12 \mathrm{~h} / 12 \mathrm{~h}$ before the experiments. In the process of modeling, $0.35 \%$ sodium pentobarbital $(35 \mathrm{mg} / \mathrm{kg}$ ) was injected into the abdominal cavity to anesthetize the animals. After the pinprick reflex and pupil reflex disappeared, the 10th lamina was surgically exposed. The spine was removed with special vascular forceps, the interspinous ligament was cut off with a micro instrument, the lamina was opened and the spinal cord was exposed. The mice were then placed on the MASCIS collimator workbench, and the 9 and 11th dorsal vertebrae were fixed. Equipped with a 10-g impact bar, the free fall from $2.5 \mathrm{~cm}$ hit the center of the spinal cord at the 10th vertebral level. It was regarded as a sign of successful modeling when the mice demonstrated compulsory convulsions or paralysis of both legs. After modeling, 20,000 units of penicillin were intramuscularly injected twice a day.

To evaluate the quality of the model, the Basso, Beattie and Bresnahan (BBB) locomotor scale $(18,19)$ was used to evaluate motor function in the mice. Motor function was divided into 22 grades, a score of 0 indicated no observable hind limb movement, a score of 21 indicated completely normal motor function, and scores between 1 and 20 were based on the level of motor function. The basic contents of reference were: The number and range of joint activity, the degree of weight bearing, the coordination of front and rear limbs and the activity of the front and rear claws and tail. Mice in the control group were fed under the same conditions as the SCI group but no treatment was included. The control and SCI groups were scored before and at $6 \mathrm{~h}, 1,3$ and 7 days after surgery.

Terminal deoxynucleotidyl-transferase-mediated dUTP nick end labeling (TUNEL) and immunofluorescence assay. The control and SCI mice were deeply anesthetized with $10 \%$ chloral hydrate $(3.5 \mathrm{ml} / \mathrm{kg}$, intraperitoneally) 3 days after surgery. $\mathrm{NaCl}$ $(0.9 \%)$ was then used to perfuse the mice, followed by $4 \%$ paraformaldehyde (PFA) in $0.01 \mathrm{M}$ phosphate-buffered saline (PBS, $\mathrm{pH}=7.4$ ). Spinal cord tissue at the region of the injury was dissected with a $0.5 \mathrm{~cm}$ margin on each side of the lesion and embedded in optimum cutting temperature (OCT) compound, after which the sections were frozen at $-80^{\circ} \mathrm{C}$, were produced. Frozen sections were rewarmed, fixed with $4 \%$ PFA for $30 \mathrm{~min}$ at room temperature, and washed 3 times with PBS. PBS containing $0.5 \%$ Triton $\mathrm{X}-100$ was added to the sections and incubated at room temperature for $5 \mathrm{~min}$. Then, the sections were washed 3 times with PBS. An appropriate amount of TUNEL detection solution (FITC-conjugated) was prepared. Sections were placed in the TUNEL detection solution, incubated at $37^{\circ} \mathrm{C}$ in the dark for $60 \mathrm{~min}$ and washed 3 times with PBS. Next, the neuron antibody NeuN (1:1,000; product code ab1777487; Abcam) was added to the sections and they were incubated at $4^{\circ} \mathrm{C}$ overnight. Cy3-conjugated secondary antibody (1:1,000; product code ab6939, Abcam) was added to the sections and they were incubated at room temperature for $2 \mathrm{~h}$. Finally, the sections were stained with Fluoroshield ${ }^{\mathrm{TM}}$ histology mounting medium (cat. no. F6057; Sigma-Aldrich; Merck KGaA) with $10 \mu \mathrm{g} / \mathrm{ml}$ 4',6-diamidino-2-phenylindole (DAPI), and sealed for microscopic observation. Imaging was performed with an Olympus FV 1000 confocal microscope (Olympus coroporation; magnification, x200). The observation area was randomly selected and no less than 5 fields were subsequently imaged.

Isolation and culture of dorsal root ganglion (DRG) neurons. WT and $\mathrm{CD}^{2} 3^{-}$mouse DRG neurons were isolated and cultured in DMEM (Gibco; Thermo Fisher Scientific, Inc.) supplemented with $10 \%$ FBS (Thermo Fisher Scientific, Inc.) in a humidified atmosphere of $5 \% \mathrm{CO}_{2}$ at $37^{\circ} \mathrm{C}$. The dorsal skin was removed under aseptic conditions with ophthalmic scissors, and then a section of the spinal cord was cut. The dorsal side was placed on a sterilized ground glass piece, the ventral half of vertebrae was cut horizontally along the two sides of the spinal canal under an anatomical microscope, the spinal cord and ganglion were exposed and the ganglion was separated with anatomical tweezers. The ganglion membrane was removed, digested with $0.125 \%$ trypsin $\left(37^{\circ} \mathrm{C}\right.$ for $\left.30 \mathrm{~min}\right)$, and then diluted into a $0.2 \times 10^{5}$ cells $/ \mathrm{ml}$ cell suspension with planting medium(DMEM and 10\% FBS). The suspension was inoculated in a 35-mm plastic culture dish coated with collagen (type I, from rat tail) which was used as a membrane coating for cell attachment, and $2 \mathrm{ml}$ of cell suspension was placed in each dish. The specimens were cultured in DMEM with $10 \%$ FBS in a $5 \% \mathrm{CO}_{2}$ incubator at $37^{\circ} \mathrm{C}$. After $24 \mathrm{~h}$, the culture medium was removed from the culture dish and replaced with fresh medium. On the third day of inoculation, the cell division inhibitors 5-fluoro-2'-deoxyuridine (15 $\mu \mathrm{g} / \mathrm{ml}$; Sigma-Aldrich; Merck KGaA) and uridine (35 $\mu \mathrm{g} / \mathrm{ml}$; Sigma-Aldrich; Merck $\mathrm{KGaA}$ ) were added to the culture dish to inhibit the proliferation of non-neural cells. After $48 \mathrm{~h}$, the fresh culture medium was changed. The medium was changed twice a week and half of the fresh culture medium was changed each time.

Hypoxia and hypoglycemia of DRG neurons. During anoxic and glucose-deficient culture, the DRG cell culture medium was aspirated, washed twice with D-Hank's solution 
(Sigma-Aldrich; Merck KGaA) and replaced with sugar-free medium. The cells were then placed into a three-gas incubator containing $1 \% \mathrm{O}_{2}, 5 \% \mathrm{CO}_{2}$ and $94 \% \mathrm{~N}_{2}$ for $0,0.5,1$ and $2 \mathrm{~h}$. After $0,0.5,1$ and $2 \mathrm{~h}$ of anoxia and glucose deficiency, the cells were removed from the three-gas incubator, the sugar-free medium was replaced with the same as that used before and the cells were placed into an ordinary cell incubator. Before anoxic and glucose deficiency treatment, cultured DRG neurons were treated with Ado and $\alpha, \beta$-methylene ADP (APCP) which is a CD73 hydrolase specific inhibitor (Sigma-Aldrich; Merck $\mathrm{KGaA}$ ). The Ado group was cultured in a medium containing an Ado final concentration of $100 \mu \mathrm{mol} / 1$ for $24 \mathrm{~h}$ at $37^{\circ} \mathrm{C}$. The APCP group was cultured in a medium containing an APCP final concentration of $10 \mu \mathrm{mol} / 1$ for $24 \mathrm{~h}$ at $37^{\circ} \mathrm{C}$. The cells of each group were collected and the related experiments were carried out.

Construction of a CD73 overexpression lentivirus. The CD73 gene CDS fragment was synthesized by Sangon Biotech Co., Ltd. and inserted into the lentivirus vector (pCDH1-MSCV-MCS1-EF1-GreenPuro Vector; System Biosciences LLC). The 2nd generation system of the lentivirus was used. Lentivirus packaging plasmids included PCDH-CD73 $(10 \mu \mathrm{g}), \operatorname{psPAX} 2(10 \mu \mathrm{g})$ and pMD2G $(10 \mu \mathrm{g})$, which were co-transfected into a $10 \mathrm{~cm}$ dish containing 293 T cells (American Type Culture Collection). Samples were transfect using the Lipofectamine ${ }^{\circledR} 2000$ transfection reagent (Thermo Fisher Scientific, Inc.). The supernatant was collected after $48 \mathrm{~h}$ of continuous culture. Appropriate amounts of PEG8000 were added to the supernatant and incubated overnight at $4^{\circ} \mathrm{C}$. The precipitate was resuspended in medium and stored at $-80^{\circ} \mathrm{C}$. Lentivirus titers were measured and used with an MOI of 10 according to the number of cells infected as needed. The isolated and cultured DRG neurons were seeded into six-well-plates $\left(5 \times 10^{4}\right.$ per well), after which $100 \mu 1$ lentivirus was added when cell confluence reached $70 \%$. Infected cells were then cultured at $37^{\circ} \mathrm{C}$ for $12 \mathrm{~h}$ and replaced with fresh medium. Cells were cultured until they were used for subsequent experimentation.

Western blotting. Tissues and cells were lysed with RIPA buffer (25 mM Tris-HCl pH 7.6, $150 \mathrm{mM} \mathrm{NaCl,} \mathrm{1 \%} \mathrm{NP-40,}$ $1 \%$ sodium deoxycholate, $0.1 \%$ SDS), and centrifuged at $4^{\circ} \mathrm{C}$ for $5 \mathrm{~min}(13,800 \mathrm{x} \mathrm{g})$, and the supernatant was collected. Total protein concentration was measured with a bicinchoninic acid (BCA) assay (Sangon Biotech Co., Ltd.). Approximately $20 \mu \mathrm{g}$ of sample was added to the loading buffer, heated at $95^{\circ} \mathrm{C}$ for 5 min, and then SDS-PAGE (12.5 and 10\% of acrylamide) electrophoresis separation was carried out. After electrophoresis, the protein samples were transferred to PVDF membranes. The CD73 antibody (1:1,000; product code ab175396; Abcam), caspase-3 antibody (1:1,000; product code ab184787; Abcam), Bcl-2 antibody (1:1,000; product code ab182858; Abcam), Bax antibody (1:1,000; product code ab182733; Abcam), Fas antibody (1:1,000; product code ab133619; Abcam), protein kinase A (PKA) antibody (1:1,000; product no. 4782S; Cell Signaling Technology, Inc.), cAMP response element-binding protein (CREB) antibody (1:1,000; product no. 9197; Cell Signaling Technology, Inc.), phosphorylated (p)-CREB antibody (1:1,000; product no. 9198; Cell Signaling Technology, Inc.) and GAPDH antibody (1:2,000; product code ab8245; Abcam) were incubated overnight at $4^{\circ} \mathrm{C}$. The membranes were subsequently blocked with $5 \%$ skimmed milk for $1 \mathrm{~h}$ at room temperature. The diluted secondary antibodies [1:2,000; cat. nos. SA00001-1 Goat anti-mouse IgG $(\mathrm{H}+\mathrm{L})$ and SA00001-2 Goat anti-rabbit $\operatorname{IgG}(\mathrm{H}+\mathrm{L})$, ProteinTech Group, Inc.] labeled with HRP was added and incubated for $2 \mathrm{~h}$ at room temperature. ECL reagent (cat. no. 34580; Thermo Fisher Scientific, Inc.) was prepared according to the manufacturer's instructions. A chemiluminescence imaging system (Tanon Science and Technology Co., Ltd.) was used to visualize the protein bands. The densitometric analysis of bands was completed using ImageJ 1.52 software (National Institutes of Health).

Detection of LDH, SOD and MDA in the cell culture supernatant and cell viability. $\mathrm{LDH}$ and MDA levels as well as SOD activity were assessed by commercialized kits (serial nos. A020-2-2, A003-4-1 and A001-3-2, respectively; Nanjing Jiancheng Bioengineering Institute). After treatment with $2 \%$ Triton $\mathrm{X}-100,100 \mu \mathrm{l}$ cell culture supernatant was extracted from each well and used to measure absorbance (A) values at $450 \mathrm{~nm}, 550 \mathrm{~nm}$ and $532 \mathrm{~nm}$ on a microplate spectrophotometer according to the instructions of the detection kit for LDH, MDA content and SOD activity. Cell viability was assessed by Cell Counting Kit-8 (CCK-8) commercialized kits (Beyotime Institute of Biotechnology). After treatment, $10 \mu \mathrm{l}$ WST-8 solution from the CCK-8 was added to each well. The absorbance value at $450 \mathrm{~nm}$ of each well was measured after $2 \mathrm{~h}$. Cell viability was calculated according to absorbance values at different time-points $(0.5,1$ and $2 \mathrm{~h})$.

Detection of intracellular ROS, ATP and cAMP content. ROS and ATP levels were measured with commercialized kits (serial nos. E004-1-1 and A095-1-1, respectively; Nanjing Jiancheng Bioengineering Institute). According to the manufacturer's instructions, the DCFH-DA probe was added to the cell medium at a final concentration of $10 \mu \mathrm{M}$. After culture for $1 \mathrm{~h}$, the culture medium was removed, cells were collected and the fluorescence intensity value of $525 \mathrm{~nm}$ was detected after $500 \mathrm{~nm}$ excitation. The value of fluorescence intensity represented the ROS content in cells. The ATP content in cells was detected by colorimetry. After the preparation of the detection reagent, the reaction was carried out according to the manufacturer's instructions. Finally, the absorbance value at $636 \mathrm{~nm}$ was detected and the ATP content was calculated according to the manufacturer's instructions. The cAMP detection kit (cat. no. 581001) was purchased from Cayman Chemical Company. The content of cAMP in the cells was detected by ELISA according to the manufacturer's instructions.

Detection of Ado in the cell culture supernatant and tissues. An Ado Assay Kit (fluorometric; product code ab211094; Abcam) was used to detect Ado in cell culture supernatant and tissues. In this assay, Ado was measured using Ado deaminase followed by a multi-step enzymatic approach resulting in the generation of an intermediate that reacts with the Ado probe, leading to the formation of a fluorescent product. The fluorescent product can be detected at ex/em $=535 / 587 \mathrm{~nm}$, and its intensity is proportional to the amount of Ado in the sample. 
A
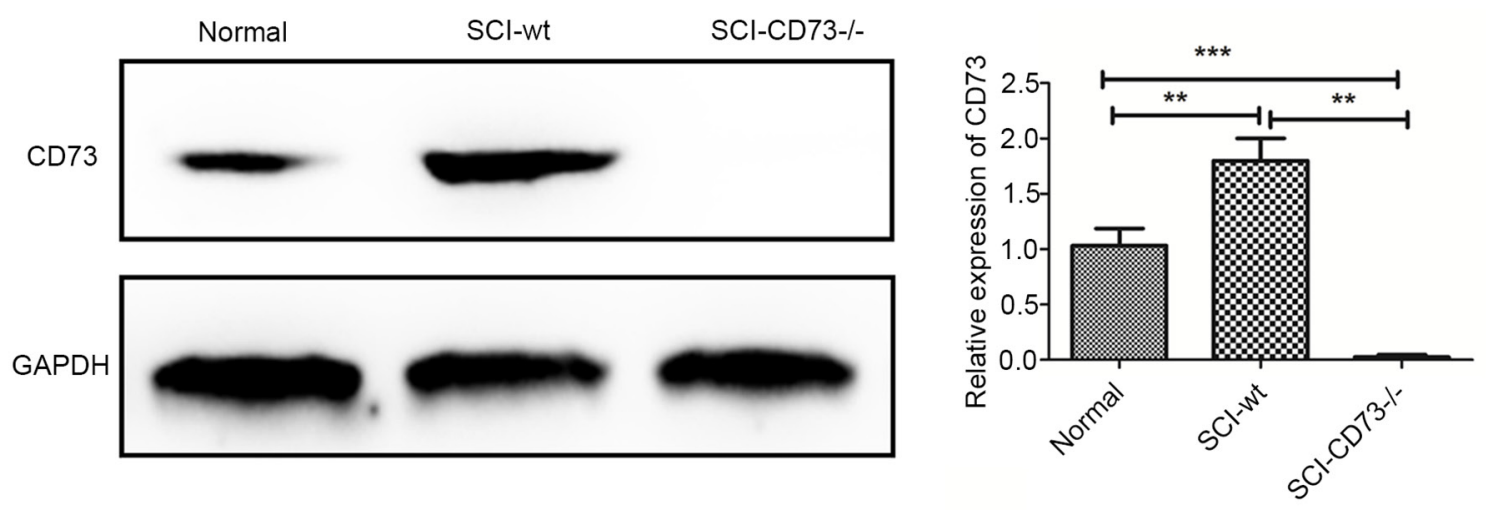

B

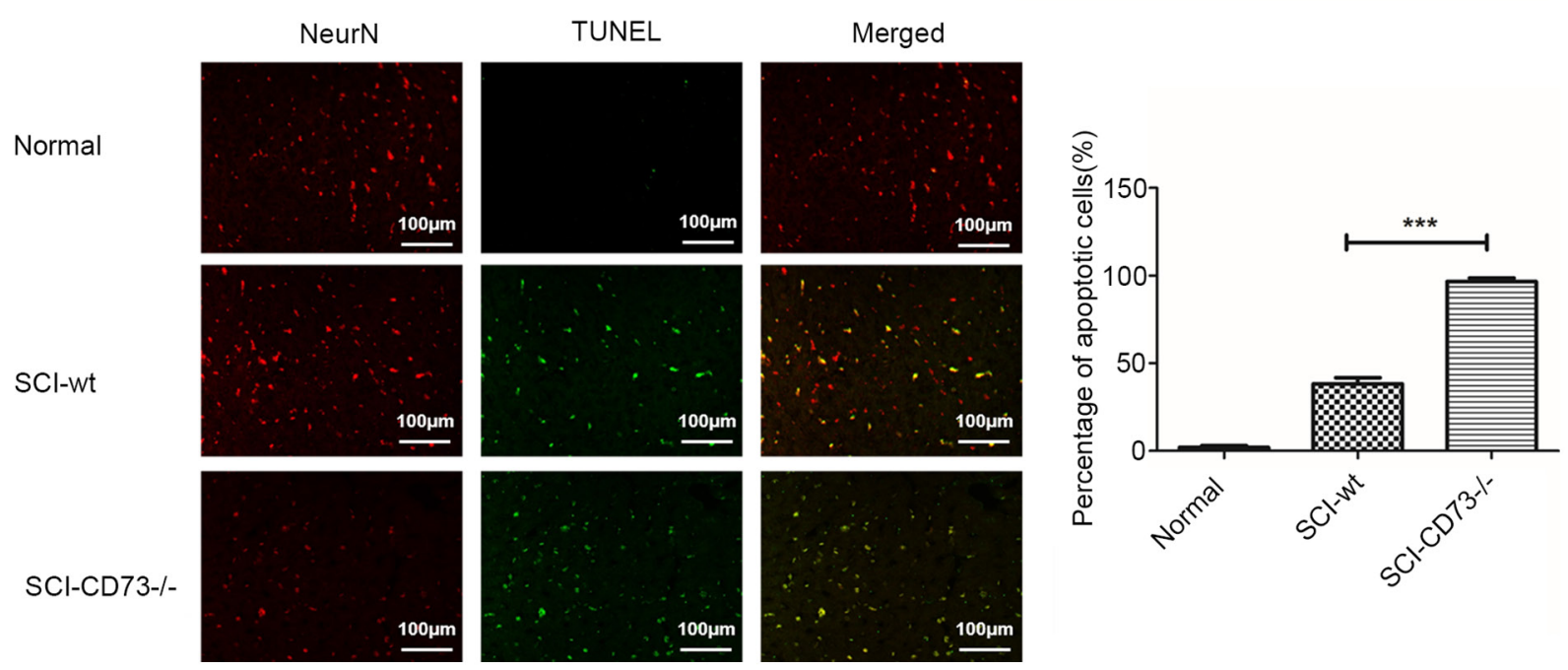

Figure 1. Neuronal apoptosis in CD73-/- mice is increased after SCI. (A) Expression of CD73 in CD73 ${ }^{-/}$and WT SCI model mice. (B) Co-staining of TUNEL and NeuN in $\mathrm{CD}_{3}{ }^{-}$and WT spinal cord injury model mice. ${ }^{* *} \mathrm{P}<0.01$ and ${ }^{* * *} \mathrm{P}<0.001$. CD73, ecto-5'-nucleotidase; SCI, spinal cord injury; WT, wild-type; TUNEL, terminal deoxynucleotidyl-transferase-mediated dUTP nick end labeling; NC, negative control.

Flow cytometry assay of apoptosis. An Annexin V-FITC Apoptosis Detection Kit (cat. no. BMS500FI-300; Thermo Fisher Scientific, Inc.) was used to evaluate apoptosis. According to manufacturer's instructions, $1 \times 10^{5}$ cultured DRG neurons were collected and washed once with PBS buffer. A total of $500 \mu \mathrm{l}$ of binding buffer were added to resuspend the cells. Then, $5 \mu 1$ Annexin V-FITC and $5 \mu 1$ propidium iodide were added to mix, and the mixture was incubated at room temperature, in the dark, for $15 \mathrm{~min}$. A flow cytometric instrument (BD FACSCalibur; BD Biosciences) was used for detection. ModFit LT 5.0 software (Verity Software House) was used for the analysis of flow cytometry data.

Statistical analysis. All results are expressed as the mean \pm standard deviation. Student's unpaired t-tests and two-way analysis of variance (ANOVA) were used to analyze data. The t-test uses the t-statistic, which is the ratio of the difference in the means to the weighted mean standard deviation, for the two sets of identical/heteroscedasticity data. ANOVA uses the F-statistic, which is the ratio of intergroup variance to intragroup variance, and is mostly used when the number of groups exceeds two. One-way ANOVA was used to examine the difference between two, three, or more groups of a dependent variable within a categorical independent variable. Two-way ANOVA was used to analyze the difference between groups of two independent variables, one of which can be regarded as a processing variable. ANOVA with Bonferroni or Dunnett's post hoc analysis was used where appropriate. $\mathrm{P}<0.05$ was considered to indicate a statistically significant difference. All statistical analyses were performed with GraphPad Prism 8.0 software (GraphPad Software, Inc.).

\section{Results}

Apoptosis of neurons from CD73\% mice is increased after spinal cord injury. To verify this hypothesis, a spinal cord injury model of $\mathrm{CD}^{-/} 3^{--}$and WT mice was constructed, the degree of injury was identified, the diseased spinal cord was stripped and transverse sections were produced. Western blot assays were performed to detect the CD73 expression level (Fig. 1A), and double fluorescence staining of TUNEL and a neuron marker (NeuN) was carried out in normal, SCI-WT and SCI-CD73 ${ }^{-1-}$ tissues (Fig. 1B). Results revealed that the apoptosis level of neurons caused by $\mathrm{SCI}$ in $\mathrm{CD} 73^{--}$mice was significantly higher than that in WT mice. Therefore, CD73 deficiency may aggravate neuronal apoptosis caused by SCI.

CD73 can inhibit neuronal apoptosis caused by oxygen-glucose deprivation. It was demonstrated that $\mathrm{CD} 73$ deficiency can increase apoptosis of neurons induced by SCI in vivo, but 
A
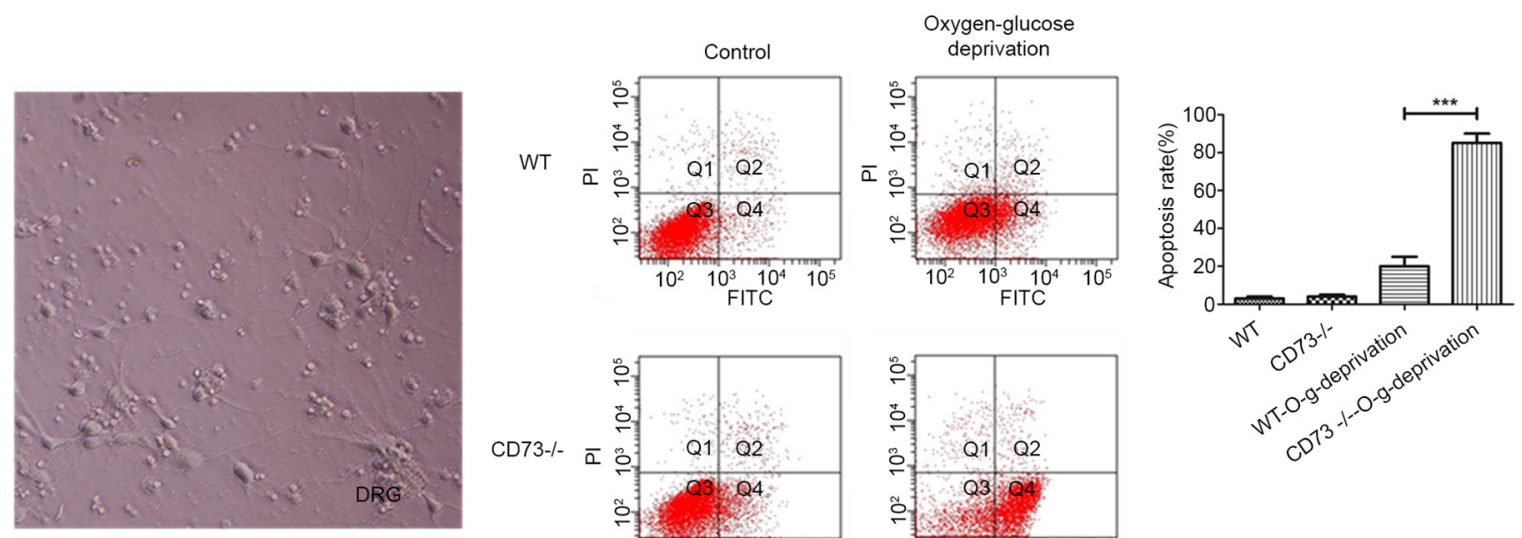

B
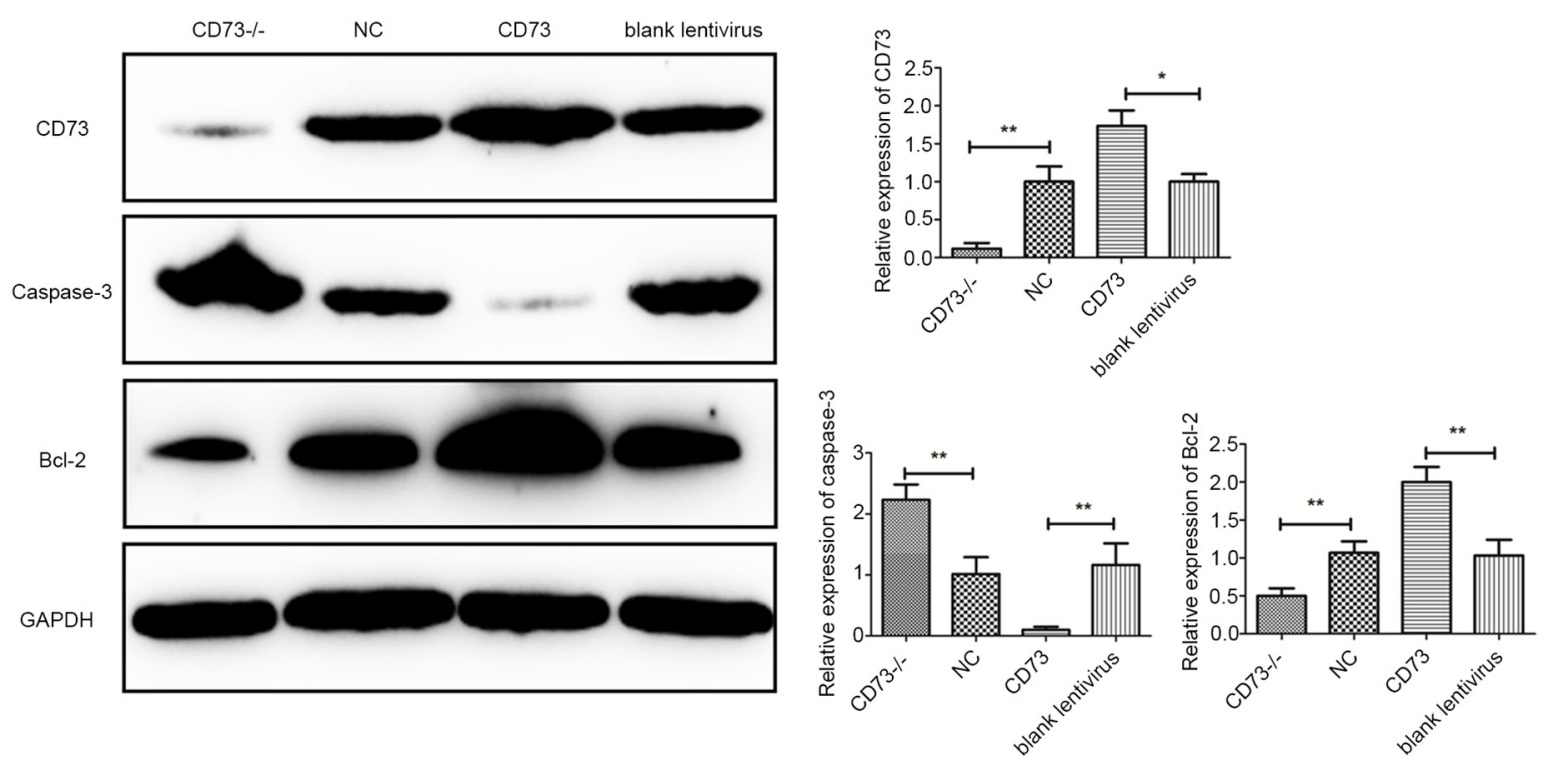

Figure 2. CD73 inhibits mouse DRG neuronal apoptosis caused by oxygen-glucose deprivation. (A) The apoptosis of CD73-/ mouse DRG neurons under oxygen-glucose deprivation were detected by flow cytometry. (B) Western blot assay was used to detect CD73, caspase-3 and Bcl-2 expression levels in CD73and CD73 mouse DRG neurons under oxygen-glucose deprivation. Blank Lentivirus, empty lentivirus-transfected cells. ${ }^{*} \mathrm{P}<0.05,{ }^{* * *} \mathrm{P}<0.01$ and ${ }^{* * *} \mathrm{P}<0.001$. CD73, ecto-5'-nucleotidase; DRG, dorsal root ganglion; NC, negative control.

whether CD73 can affect apoptosis of normal spinal cord neurons in vitro has not been confirmed. Therefore, DRG neurons from the spinal cord of $\mathrm{CD} 73^{-/}$and WT mice were isolated and cultured. The apoptosis of both $\mathrm{CD} 73^{-/}$and WT DRG neurons was detected by flow cytometry after anoxia and glucose deficiency treatment (Fig. 2A). Results revealed that the apoptosis of $\mathrm{CD} 73^{-/}$mouse DRG neurons increased under anoxic and glucose-deficient conditions. To further study the relationship between CD73 and neuronal apoptosis, a CD73 overexpression lentivirus was used to enhance the CD73 expression level in DRG neurons before treatment with oxygen-glucose deprivation. Then the expression levels of CD73, an apoptosis gene (caspase-3) and an antiapoptotic gene (Bcl-2) in the CD73 group (CD73 overexpression group), CD73 ${ }^{-/}$group, blank lentivirus group and $\mathrm{NC}$ group were detected by western blotting (Fig. 2B). The results indicated that the expression level of CD73 was negatively associated with that of caspase-3 and positively associated with the expression level of Bcl-2.
To study the effects of CD73 on the normal physiological function of DRG neurons, LDH, MDA, SOD contents in cell culture medium and ATP, ROS and cell viability in cells were measured in each group (Fig. 3). Compared with the NC group, the release of $\mathrm{LDH}$, and MDA and the production of ROS in $\mathrm{CD} 73^{-/}$DRG neurons was increased, while the synthesis of ATP, SOD and cell viability was decreased after hypoxia-hypoglycemia treatment. However, the CD73-overexpression group revealed the opposite effects. These results demonstrated that CD73 could inhibit the apoptosis of neurons caused by glucose deficiency and hypoxia and improve the adaptability of neurons to harsh environments.

CD73 decreases neuronal apoptosis by promoting Ado synthesis. Firstly, the relationship between CD73 and Ado was examined. The extracellular Ado concentration in DRG neurons was significantly decreased after CD73 was knocked out, while it was significantly increased after CD73 was 

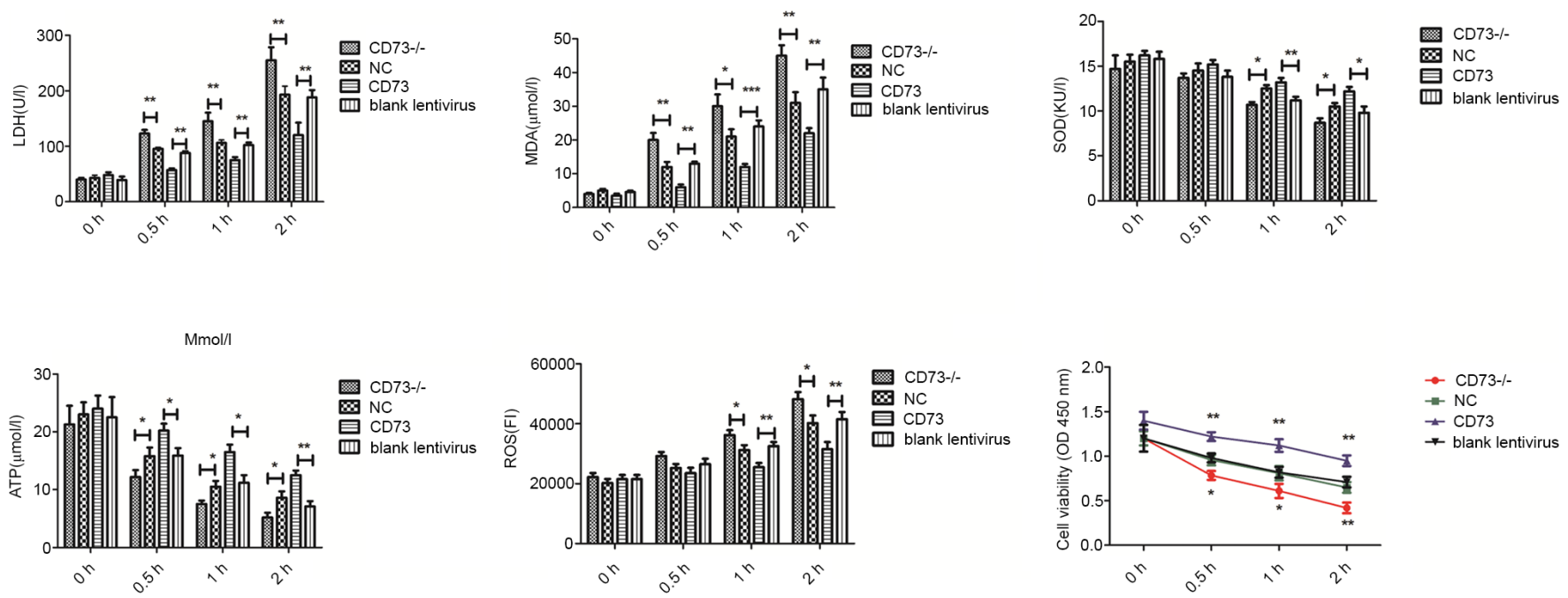

Figure 3. LDH, MDA, SOD contents in cell culture medium and ATP, ROS and cell viability in CD73 ${ }^{-/}$and CD73 mouse dorsal root ganglion neurons under oxygen-glucose deprivation. The treatment time-points were $0,0.5,1$ and $2 \mathrm{~h}$. Blank Lentivirus, empty lentivirus-transfected cells. ${ }^{*} \mathrm{P}<0.05,{ }^{* *} \mathrm{P}<0.01$ and ${ }_{* * * *} \mathrm{P}<0.001$. LDH, lactate dehydrogenase; MDA, malondialdehyde; SOD, superoxide dismutase; ATP, adenosine triphosphate; ROS, reactive oxygen species; CD73, ecto-5'-nucleotidase; NC, negative control.

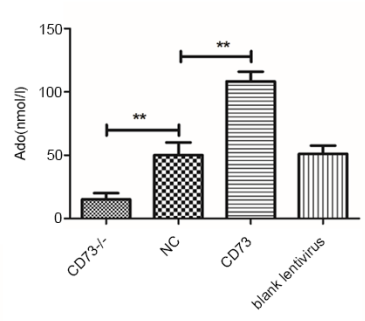

B

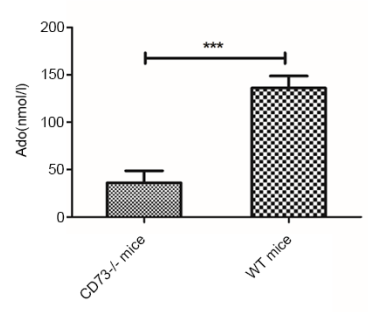

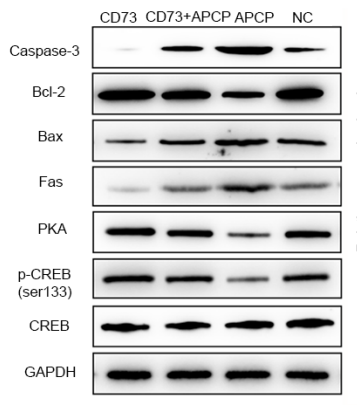

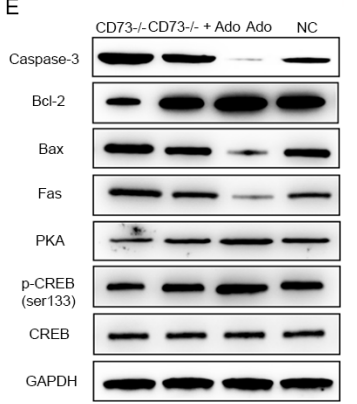

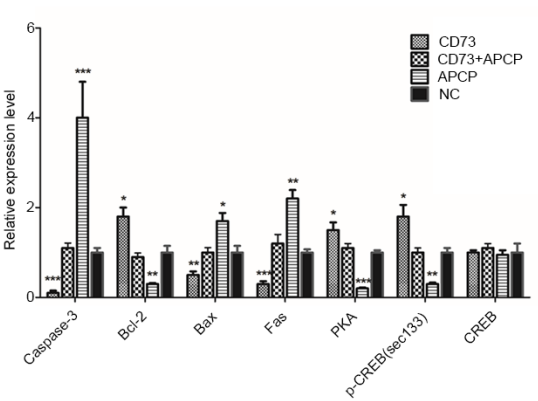

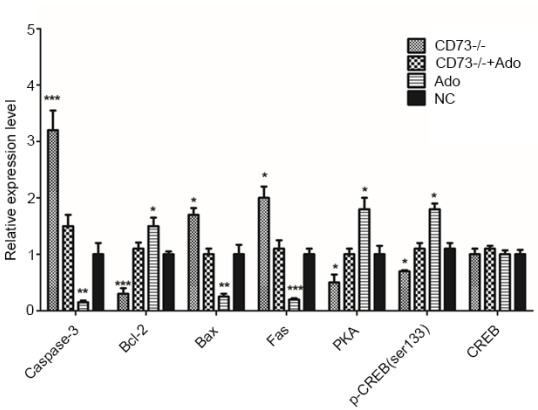

D

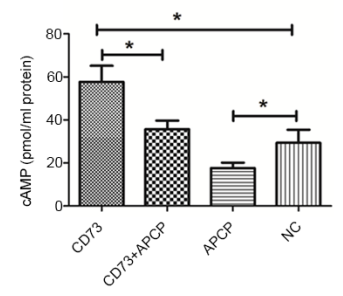

$\mathrm{F}$

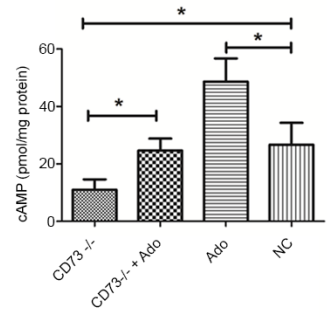

Figure 4. Effects of CD73, APCP and Ado on DRG neurons in hypoxic and hypoglycemic environments. (A) The extracellular Ado concentration in CD73 ${ }^{-1}$ and CD73 DRG neurons. (B) The Ado concentration in the spinal cord of CD73 $3^{-1}$ and WT mice. (C) The expression of apoptosis-related genes and PKA/ CREB signaling pathway genes in CD73- and APCP-treated mouse DRG neurons under oxygen-glucose deprivation. (D) The content of cAMP in CD73- and APCP-treated mouse DRG neurons under oxygen-glucose deprivation. (E) The expression of apoptosis-related genes and PKA/CREB signaling pathway genes in $\mathrm{CD}^{-/} 3^{-}$- and Ado-treated mouse DRG neurons under oxygen-glucose deprivation. (F) The content of cAMP in CD73 $3^{--}$and Ado-treated mouse DRG neurons under oxygen-glucose deprivation. ${ }^{*} \mathrm{P}<0.05,{ }^{* *} \mathrm{P}<0.01$ and ${ }^{* * * *} \mathrm{P}<0.001$. CD73, ecto-5'-nucleotidase; APCP, $\alpha, \beta$-methylene ADP; Ado, adenosine; DRG, dorsal root ganglion; WT, wild-type; PKA/CREB, protein kinase A/cAMP response element-binding protein; NC, negative control.

overexpressed (Fig. 4A). Similarly, Ado concentration levels in the spinal cord of $\mathrm{CD} 73^{--}$mice were significantly lower than those of control WT mice (Fig. 4B). These results indicated that the expression level of CD73 was positively associated with the concentration of Ado. Whether the protective effect of CD73 on neurons is related to its metabolic Ado function is unclear. Therefore, DRG neurons were treated with APCP and Ado to inhibit and enhance the CD73-mediated Ado synthesis pathway before low glucose and hypoxia treatment. Then western blot analysis was performed to detect the expression of apoptosis-related proteins in neurons (Fig. 4C and E). The results demonstrated that both blocking the Ado synthesis pathway with APCP and knocking out CD73 could increase the neuronal apoptosis induced by glucose deficiency and hypoxia. Proapoptotic genes such as those of the death receptor pathway (Fas) and the mitochondrial pathway (Bax 


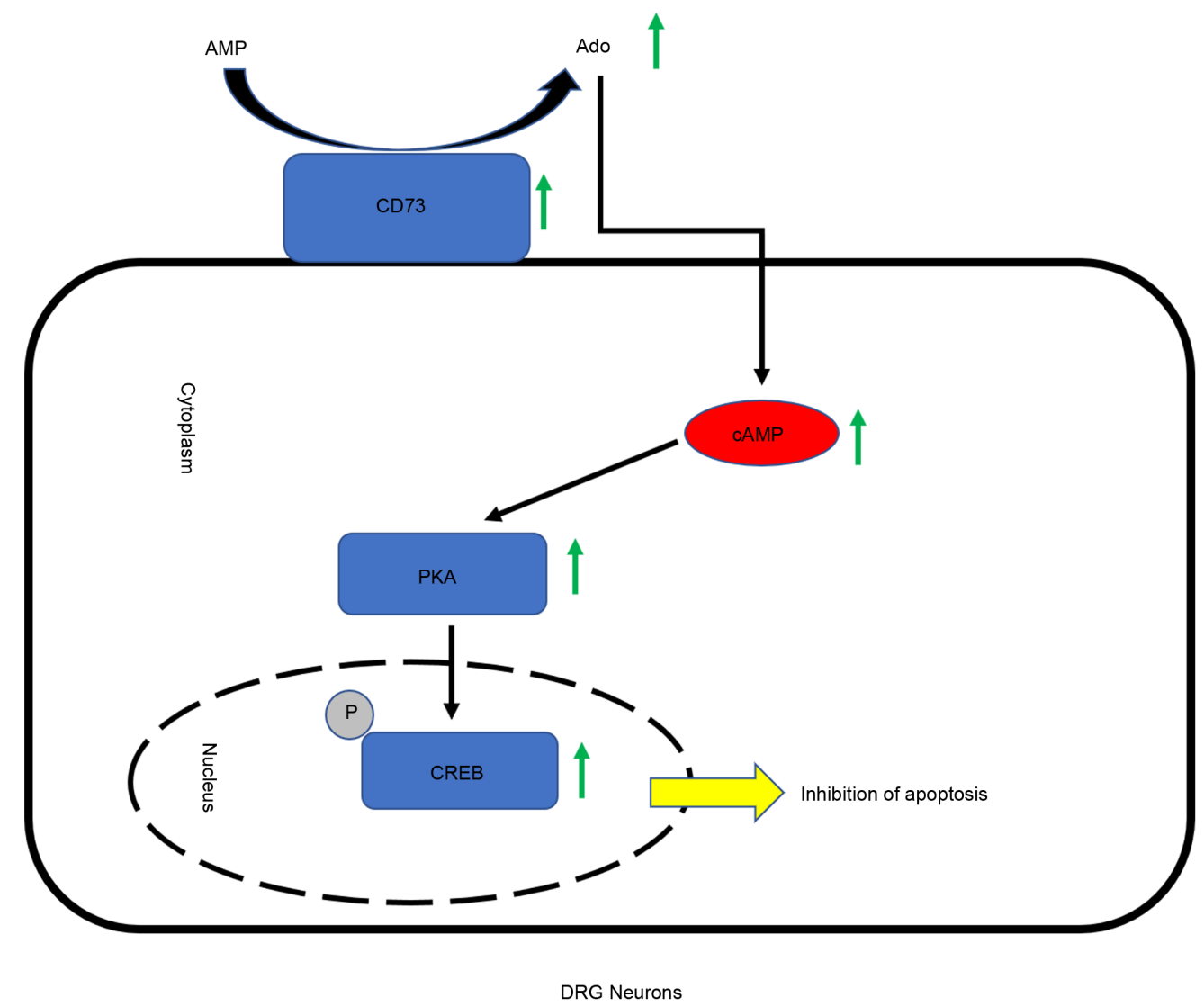

Figure 5. Schematic diagram of the protective mechanism of CD73 in dorsal root ganglion neurons. CD73, ecto-5'-nucleotidase; PKA, protein kinase A; CREB, cAMP response element-binding protein; Ado, adenosine.

and caspase-3) were both induced, while antiapoptotic gene Bcl-2 was decreased. However, this effect could be reversed by overexpressing the CD73 gene or adding Ado to the culture medium. Thus, it was demonstrated that CD73 could protect spinal cord neurons from external hypoxia and a low glucose environment by influencing Ado metabolism.

CD73 activates the $c A M P / P K A / C R E B$ pathway by enhancing Ado synthesis. A previous study has revealed that Ado is closely related to CAMP which has been shown to protect damaged neurons by activating PKA and CREB (20). Therefore, whether CD73 affects the cAMP/PKA/CREB signaling pathways by regulating Ado synthesis was investigated. Western blot assays and cAMP content detection were performed to verify the relationship of $\mathrm{CD} 73$, Ado and the $\mathrm{cAMP} / \mathrm{PKA} / \mathrm{CREB}$ signaling pathway (Fig. 4C-F). Results indicated that the intracellular content of cAMP and the expression of PKA and p-CREB were upregulated by CD73 overexpression or Ado but downregulated by $\mathrm{CD} 73^{-/}$and APCP. In summary, CD73 could activate the $\mathrm{CAMP} / \mathrm{PKA} / \mathrm{CREB}$ signaling pathway by enhancing Ado synthesis.

\section{Discussion}

It was observed in a previous study that CD73 deficiency exacerbated the motor dysfunction, inflammatory responses, and neuronal apoptosis induced by SCI (17). Therefore, focus was directed on the neuroprotective effect of CD73 in SCI.
Firstly, SCI models with $\mathrm{CD} 73^{-/-}$mice and WT mice were constructed. Subsequently, immunofluorescence and TUNEL staining assays revealed that the apoptosis level of spinal cord neurons in $\mathrm{CD} 73^{-/}$SCI mice was significantly higher than that in the control group. Results indicated that CD73 may inhibit neuronal apoptosis caused by SCI in vivo. To further investigate the relationship between CD73 and neuronal apoptosis, CD73-/-, CD73 and blank lentivirus group neurons and NC spinal DRG neurons were cultured before anoxia and glucose deficiency treatment. It was demonstrated that CD73 could inhibit the apoptosis of DRG neurons, suppress the release of LDH, MDA and ROS, and increase the synthesis of ATP, SOD and cell viability caused by glucose deficiency and hypoxia. CD73 was revealed to protect neurons both in vitro and in vivo, and its mechanism is worthy of further study.

CD73 is a multifunctional extracellular nuclease, an enzyme that mainly catalyzes AMP to Ado. Numerous studies have confirmed that Ado, as an important molecule in the process of nucleotide metabolism, has a clear protective effect on ischemic neurons (21-25). Therefore, the initiation of the endogenous adenosine pathway has become an important candidate for the protection of ischemic neurons. The cAMP molecule, which is regulated by Ado, is an important second messenger of intracellular signal transduction (20). PKA, which is cAMP-dependent, is the classic signal transduction pathway (26). The activated PKA catalytic subunit can enter the nucleus and regulate cell metabolism and gene expression by catalyzing the phosphorylation of serine or threonine 
residues of proteins in the cell, the most famous of which is CREB $(27,28)$. Apoptosis induced by ischemia and hypoxia can be initiated by various endogenous and exogenous signals, which are ultimately regulated by genes. In this process, caspases of cysteine are an important intracellular initiating mechanism for apoptosis (29), and Bcl-2 can participate in the regulation of this caspase pathway, thus indirectly affecting the occurrence of apoptosis in disease (30). Meller et al reported that phosphate-acidified CREB after the prestimulation of ischemia could mediate the expression of the antiapoptotic gene Bcl-2, which could reduce the apoptosis of cells and thus play a role in protecting nerve cells (31). Shieh et al reported that the phosphorylation of CREB activated the expression of brain-derived neurotrophic factor (BDNF) which is widely distributed in the central nervous system, playing an important role in the survival, differentiation, growth and development of neurons, preventing the death of damaged neurons, improving the pathological state of neurons, and promoting the regeneration and differentiation of injured neurons and other biological effects (32). Therefore, it was hypothesized that CD73 could affect Ado synthesis and then indirectly affect the content of intracellular cAMP, and the increased cAMP could activate the PKA/CREB signaling pathway, thereby inhibiting cell apoptosis.

To verify this hypothesis, a series of in vitro cell experiments was conducted and the results demonstrated that CD73 and Ado could inhibit the expression of proapoptotic genes (Fas, Bax and caspase-3) and induce an antiapoptotic gene (Bcl-2) when neurons encountered a hostile environment. In addition, CD73 and Ado also promoted the synthesis of cAMP and activated the cAMP/PKA/CREB signaling pathway. The molecular mechanism of CD73 inhibition of DRG neuronal apoptosis can be described as follows: When DRG neurons encounter harsh living conditions, highly expressed CD73 can generate more Ado, which can promote an increase in intracellular cAMP content. PKA can be activated and promote the phosphorylation level of CREB. p-CREB subsequently activates a number of signaling pathways to reduce the apoptosis caused by the harsh environment and improve the adaptability of neurons (Fig. 5).

In conclusion, it was revealed that CD73 activated the cAMP/PKA/CREB signaling pathway by increasing Ado synthesis to protect neurons from apoptosis. This finding will deepen the understanding of the neuroprotective mechanism of CD73 and Ado, and provide a new theoretical basis for the treatment of SCI and other neurological injuries in the future. However, the specific molecular mechanism by which CD73 inhibits neuronal apoptosis through signaling pathways and whether CD73 has other ways to affect neuronal apoptosis remain to be further investigated. In the future, more CD73-regulated genes and signaling pathways will be studied through high-throughput sequencing technology. Through the in-depth study of them, it is anticipated to deepen the understanding of the function of CD73 and provide novel insights into the treatment of diseases related to neuronal apoptosis.

\section{Acknowledgements}

Not applicable.

\section{Funding}

The present study was supported by the National Natural Science Foundation of China (grant no. 81871552) and the National Science Foundation for Distinguished Young Scholars of China (grant no. 81802145).

\section{Availability of data and materials}

The datasets used and/or analyzed during the current study are available from the corresponding author on reasonable request.

\section{Authors' contributions}

MS constructed the animal model and performed DRG cell isolation and culture, performed lentivirus cell infection, analyzed the data and wrote the original draft of the manuscript. CZ performed the western blotting, conducted the flow cytometry assay and analyzed the data. XM performed the remaining experiments and contributed to the writing, reviewing and editing of the manuscript. FL designed, conceived and supervised the study. MS and FL confirm the authenticity of all the raw data. All authors read and approved the manuscript and agree to be accountable for all aspects of the research in ensuring that the accuracy or integrity of any part of the work are appropriately investigated and resolved.

\section{Ethics approval and consent to participate}

All surgical procedures and experimental protocols in the present study were performed in accordance with standard guidelines approved by the Ethics Committee of Experimental Research, Shanghai Medical College, Fudan University (Shanghai, China). The approval no. is 2019-Huashan Hospital-JS-104.

\section{Patient consent for publication}

Not applicable.

\section{Competing interests}

The authors declare that they have no competing interests.

\section{References}

1. Eckert MJ and Martin MJ: Trauma: Spinal cord injury. Surg Clin North Am 97: 1031-1045, 2017.

2. Li J, Liu G, Zheng Y, Hao C, Zhang Y, Wei B, Zhou H and Wang D: The epidemiological survey of acute traumatic spinal cord injury (ATSCI) of 2002 in Beijing municipality. Spinal Cord 49: 777-782, 2011.

3. Ning GZ, Yu TQ, Feng SQ, Zhou XH, Ban DX, Liu Y and Jiao XX: Epidemiology of traumatic spinal cord injury in Tianjin, China. Spinal Cord 49: 386-390, 2011.

4. Chang FS, Zhang Q, Sun M, Yu HJ, Hu LJ, Wu JH, Chen G, Xue LD and Lu J: Epidemiological study of spinal cord injury individuals from halfway houses in Shanghai, China. J Spinal Cord Med 41: 450-458, 2018.

5. Rabchevsky AG, Patel SP and Springer JE: Pharmacological interventions for spinal cord injury: Where do we stand? How might we step forward? Pharmacol Ther 132: 15-29, 2011. 
6. Hou JM, Sun TS, Xiang ZM, Zhang JZ, Zhang ZC, Zhao M, Zhong JF, Liu J, Zhang H, Liu HL, et al: Alterations of resting-state regional and network-level neural function after acute spinal cord injury. Neuroscience 277: 446-454, 2014

7. Hurlbert RJ: Methylprednisolone for the treatment of acute spinal cord injury: Point. Neurosurgery 61 (Suppl 1): S32-S35, 2014.

8. Oyinbo CA: Secondary injury mechanisms in traumatic spinal cord injury: A nugget of this multiply cascade. Acta Neurobiol Exp (Wars) 71: 281-299, 2011.

9. Balsam LB: Spinal cord ischemia-reperfusion injury: MicroRNAs and mitophagy at a crossroads. J Thorac Cardiovasc Surg 154: 1509-1510, 2017.

10. Knoblach SM, Huang X, VanGelderen J, Calva-Cerqueira D and Faden AI: Selective caspase activation may contribute to neurological dysfunction after experimental spinal cord trauma. J Neurosci Res 80: 369-380, 2005.

11. Nardone R, Pikija S, Mutzenbach JS, Seidl M, Leis S, Trinka E and Sellner J: Current and emerging treatment options for spinal cord ischemia. Drug Discov Today 21: 1632-1641, 2016.

12. Xu W, Chi L, Xu R, Ke Y, Luo C, Cai J, Qiu M, Gozal D and Liu R: Increased production of reactive oxygen species contributes to motor neuron death in a compression mouse model of spinal cord injury. Spinal Cord 43: 204-213, 2005.

13. Antonioli L, Pacher P, Vizi ES and Haskó G: CD39 and CD73 in immunity and inflammation. Trends Mol Med 19: 355-367, 2013

14. Kulesskaya N, Võikar V, Peltola M, Yegutkin GG, Salmi M, Jalkanen S and Rauvala H: CD73 is a major regulator of adenosinergic signalling in mouse brain. PLoS One 8: e66896, 2013.

15. Tsutsui S, Schnermann J, Noorbakhsh F, Henry S, Yong V, Winston BW, Warren K and Power C: A1 adenosine receptor upregulation and activation attenuates neuroinflammation and demyelination in a model of multiple sclerosis. J Neurosci 24 1521-1529, 2004

16. Lee JY, Jhun BS, Oh YT, Lee JH, Choe W, Baik HH, Ha J, Yoon KS, Kim SS and Kang I: Activation of adenosine A3 receptor suppresses lipopolysaccharide-induced TNF-alpha production through inhibition of PI 3-kinase/Akt and NF-kappaB activation in murine BV2 microglial cells. Neurosci Lett 396: $1-6,2006$.

17. Xu S, Zhu W, Shao M, Zhang F, Guo J, Xu H, Jiang J, Ma X, Xia X, Zhi X, et al: Ecto-5'-nucleotidase (CD73) attenuates inflammation after spinal cord injury by promoting macrophages/microglia M2 polarization in mice. J Neuroinflammation 15: 155, 2018.

18. Basso DM, Beattie MS and Bresnahan JC: A sensitive and reliable locomotor rating scale for open field testing in rats. J Neurotrauma 12: 1-21, 1995.

19. Basso DM, Beattie MS and Bresnahan JC: Graded histological and locomotor outcomes after spinal cord contusion using the NYU weight-drop device versus transection. Exp Neurol 139: 244-256, 1996.
20. Gerlo S, Kooijman R, Beck IM, Kolmus K, Spooren A and Haegeman G: Cyclic AMP: A selective modulator of NF- $\kappa B$ action. Cell Mol Life Sci 68: 3823-3841, 2011.

21. Kitagawa H, Mori A, Shimada J, Mitsumoto Y and Kikuchi T: Intracerebral adenosine infusion improves neurological outcome after transient focal ischemia in rats. Neurol Res 24: 317-323, 2002.

22. Gervitz LM, Nalbant D, Williams SC and Fowler JC: Adenosine-mediated activation of Akt/protein kinase B in the rat hippocampus in vitro and in vivo. Neurosci Lett 328: 175-179, 2002.

23. Zalewska-Kaszubska J: Neuroprotective mechanisms of adenosine action on CNS neurons. Neurol Neurochir Pol 36: 329-336, 2002 (In Polish).

24. Wan Q, Zhuang WF, Yao H, Liu ZW, Huang YH and Ding AS: Effects of adenosine on intracellular free calcium in cultured rat hippocampal CA1 neurons during anoxia. Sheng Li Xue Bao 49: 545-550, 1997 (In Chinese).

25. Wan Q, Yao H and Wang F: Involvement of $\mathrm{K}(+)$ channels in the inhibitory effects of adenosine on anoxia-induced $[\mathrm{Ca}(2+)](\mathrm{i})$ increase in cultured rat hippocampal CA1 neurons. Biol Signals Recept 8: 309-315, 1999.

26. Walsh DA, Perkins JP and Krebs EG: An adenosine 3',5'-monophosphate-dependant protein kinase from rabbit skeletal muscle. J Biol Chem 243: 3763-3765, 1968.

27. Mayr B and Montminy M: Transcriptional regulation by the phosphorylation-dependent factor CREB. Nat Rev Mol Cell Biol 2: 599-609, 2001.

28. Montminy MR, Gonzalez GA and Yamamoto KK: Regulation of cAMP-inducible genes by CREB. Trends Neurosci 13: 184-188, 1990.

29. Zhang H, Li Q, Li Z, Mei Y and Guo Y: The protection of $\mathrm{Bcl}-2$ overexpression on rat cortical neuronal injury caused by analogous ischemia/reperfusion in vitro. Neurosci Res 62: 140-146, 2008

30. Ruffolo SC and Shore GC: BCL-2 selectively interacts with the BID-induced open conformer of BAK, inhibiting BAK auto-oligomerization. J Biol Chem 278: 25039-25045, 2003.

31. Meller R, Minami M, Cameron JA, Impey S, Chen D, Lan JQ, Henshall DC and Simon RP: CREB-mediated Bcl-2 protein expression after ischemic preconditioning. J Cereb Blood Flow Metab 25: 234-246, 2005.

32. Shieh PB, Hu SC, Bobb K, Timmusk T and Ghosh A: Identification of a signaling pathway involved in calcium regulation of BDNF expression. Neuron 20: 727-740, 1998.

This work is licensed under a Creative Commons Attribution-NonCommercial-NoDerivatives 4.0 International (CC BY-NC-ND 4.0) License. 